\title{
marges Marges
}

revue d'art contemporain Revue d'art contemporain

19 | 2014

Les temps de l'art

\section{Les temps de l'art : une introduction}

\section{Stéphane Reboul et Umut Ungan}

\section{(2) OpenEdition}

\section{Journals}

Édition électronique

URL : http://journals.openedition.org/marges/937

DOI : 10.4000/marges.937

ISSN : 2416-8742

\section{Éditeur}

Presses universitaires de Vincennes

\section{Édition imprimée}

Date de publication : 1 octobre 2014

Pagination : 11-16

ISBN : 978-2-84292-415-7

ISSN : $1767-7114$

Référence électronique

Stéphane Reboul et Umut Ungan, «Les temps de l'art : une introduction », Marges [En ligne], 19 | 2014, mis en ligne le 01 octobre 2016, consulté le 07 mai 2019. URL : http://journals.openedition.org/ marges/937 ; DOI : 10.4000/marges.937 


\section{Les temps de l'art: une introduction}

Tout en ayant conscience des risques interprétatifs - de surinterprétation - et de réduction conceptuelle que pouvait engendrer une notion aussi étendue, "flottante» et instable que les «temps de l'art», nous pensons néanmoins que cette expression permet de renouveler la réflexion sur la diversité des modes temporels dans les arts. Pour cela, nous avons élaboré dans notre appel un certain nombre de cadres qui donnent des orientations ouvertes de recherche tout en délimitant leurs champs d'investigation; les orientations pragmatiques de ces cadres permettent d'envisager des rencontres interprétatives "vertueuses ». La sélection des textes retenus, confirme l'émergence de questionnements différents qui néanmoins partagent des axes communs d'analyse animés par une exigence pragmatique, pouvant se recouper et s'enrichir mutuellement. L'intérêt de proposer des «mailles conceptuelles » assez larges fut de favoriser des rencontres moins attendues entre des auteurs ayant des centres d'intérêt, des recherches et des disciplines différentes. L'un des succès de cette journée d'étude est d'avoir suscité des débats animés entre les participants (intervenant ou non).

L'utilisation d'un pluriel pour « le(s) temps de l'art » a permis d'interroger et d'explorer la diversité des modalités temporelles dans les arts. Un des enjeux de cette pluralité des temps de l'art est de concevoir une plus grande coopération entre l'artistique et l'esthétique/1. L'artistique n'est pas seulement ce qui intervient en amont du monde de l'art et l'esthétique ce qui apparaitrait en aval; Mais chacun, tout en ayant
/1 Voir la notion «d'immanence plurielle» et plus particulièrement d'« œuvre plurielle » de Gérard Genette qu'il définit comme le fait que l'œuvre agit diversement selon les lieux, les époques, les individus, les circonstances. L'œuvre excède son immanence en vertu d'une pluralité opérale qui est d'ordre attentionnel: « c'est ici l'œuvre, comme objet de réception et de relation esthétique, qui revêt, selon les circonstances et les contextes, des allures et des significations différentes. » Gérard Genette, L'œuvre de l'art: Immanence et Transcendance, Paris, Seuil, 1994, p. 259. 
/ 2 Jean Marie Schaeffer, Adieu à l'esthétique, Paris, PUF, 2000, p. 44. L'hypothèse est d'envisager non seulement des interactions entre un passé, un présent et un future de l'œuvre - par exemple entre ses procès de conception, de production et de réception - mais de concevoir aussi que celles-ci puissent agir tant de manière diachronique, synchronique que rétroactive.

/3 Voir Pierre Livet, Qu'est-ce qu'une action? Vrin, 2005; id "La perception de l'action » dans id. (sld), De la perception à l'action, Paris, Vrin, 2000.

/4 Pierre Livet, Qu'est-ce qu'une action?, op. cit., p. 21-22.

15 ibid. son propre temps relativement autonome, peut agir sur l'autre sans aucune hiérarchie causale et logique : en effet, même s' « il n'existe pas de dépendance logique entre les deux activités/2 ", la question de la pluralité des temps d'une œuvre permet une réflexion ouverte et riche sur les relations qui existent entre les sphères artistique et esthétique. Les temps de la conception, de la production d'une œuvre se nourrissent des expériences esthétiques de l'artiste ; tout comme l'esthéticien participe au temps de l'œuvre au moment de sa réception.

Un deuxième enjeu, ou plutôt une des conséquences pragmatiques d'un «pluralisme temporel » pour une œuvre d'art, serait qu'elle s’engage pour un "réalisme modéré » permettant de déconstruire l'opposition métaphysique entre réalisme et idéalisme. Ces œuvres à temporalité plurielle ont une « objectalité » et une «objectivité » relatives où leur(s) contexte(s) joue(nt) un rôle déterminant : leur réalisme passe par une montée en objectivité qui s'élabore à partir des subjectivités des actions des différents agents du monde de l'art.

\section{Deux approches interactives du temps : action et discours temporels chez Pierre Livet et Hans Belting}

Cela revient aussi à concevoir l'action d'une œuvre d'art comme une coopération entre une diversité d'agents. Dans ce sens, un premier cadre théorique de cette approche peut être la notion d'action de Pierre Livet/3. Il s'agirait pour nous d'associer de manière pragmatique les notions d'action et de temporalité: quelles sont les différentes actions de ces temps pluriels d'une œuvre d'art? On peut considérer le temps comme une action, au moins dans le sens où il est une de ses conditions de possibilité, ce qui conduit l'action.

Pierre Livet remet en cause le schéma linéaire de l'action qui conçoit celle-ci comme une suite de mouvements animés par une intention. Il n'est pas nécessaire qu'une intention consciente précède l'action, ni qu'une intention soit consciente pendant l'action. Ces impasses tiennent à ce que "l'intention n'est pas le commencement de nos actions, c'en est le résultat/4 ". Une condition des intentions est de disposer d'une vaste gamme de mouvements variés afin d'opérer des comparaisons. La genèse des capacités intentionnelles de l'action passe par des ramifications, des arborescences afin de constituer un riche réservoir de variations possibles, autant vers le futur de l'action que vers ses pré-conditions. "À une séquence linéaire se substituent des réseaux de reconnexion entre deux arborescences en aval et en amont, selon une dynamique de l'action qui réenrichit et reconstitue ses propres bases au fur et à mesure qu'elle se développe/5. » 
Dès lors, l'action du temps de l'œuvre peut aussi faire l'objet d'une remise en cause de son fonctionnement linéaire. À partir de là, on peut concevoir un schéma non linéaire de l'action dans et entre les procès de conception, de production et de réception d'une œuvre d'art. Notre hypothèse est d'envisager non seulement des interactions entre un passé, un présent et un futur de l'œuvre - par exemple entre ses procès de conception, de production et de réception - mais de concevoir aussi que celles-ci puissent agir autant de manière diachronique, synchronique que rétroactive.

Cette action temporelle des œuvres à temporalité plurielle permet aussi de déconstruire l'opposition entre poïesis et praxis qui chez Pierre Livet passe par les notions d'exploration et d'exploitation/6. On redonne aux activités praxiques leur rareté et à celle poḯtiques leur fréquence, en interrogeant sur ce qu'impliquent les possibilités de révision. Dans l'utilisation des capacités de révision pour reconverger vers le noyau de l'action, celles-ci peuvent assurer des articulations avec d'autres actions. Ce procédé conduit à relever qu'à toute étape de l'action il se réserve des branchements pour d'autres actions possibles. Dès lors, on peut distinguer deux tendances de nos activités. Celles consistant à poursuivre ces branchements sans se soucier de revenir au noyau de l'action initiale : une activité d'exploration et celle consistant à toujours renouer avec le noyau de l'action première, à savoir d'atteindre la cible et la satisfaction de la motivation: une activité d'exploitation et d'utilisation.

Pierre Livet nous montre qu'il n'est pas possible de séparer radicalement la praxis de la poiésis, l'exploration de l'utilisation. « Nos productions nous permettent de nous donner des repères pour nos actions, et de définir des noyaux d'action. Nos révisions nous permettent de combiner une action avec d'autres, et d'ouvrir un ensemble d'actions sur une extension illimitée de leur réseau. Les productions ou les satisfactions qui arrêtent les actions nous permettent de nous y retrouver dans ce réseau et de définir des catégories d'usages. Nous ne pouvons développer la praxis ou l'exploitation sans avoir développé la poiésis ou la production, et inversement/7. " Cette action non linéaire du temps qui permet une coopération entre praxis et poiesis relève de ce «temps artistique pluriel » présenté par les auteurs et qui nous montrent aussi un mode de résistance au «temps technologique » qui, sous prétexte d'efficacité, limite nos capacités à se l'approprier, à l'actualiser, en imposant en fait un usage unique pour ces temps technologiques. Il y a donc aussi une " théâtralité de la temporalité » qui impose un usage unique du temps et pour lesquels une œuvre se développant dans des temps pluriels, constituerait une alternative libertaire: le temps artistique
/6 Rappelons la distinction d'Aristote qui reste à la base des analyses de Pierre Livet :

1) La poiésis est une action en vue d'une fin qui est différente d'elle-même, alors que la praxis n'est pas faite en vue d'autre chose.

2) La poiésis se termine par un résultat qui se détache de l'action. Le résultat obtenu par la praxis est elle-même. 3) On ne peut pas avoir eu une action poiétique (donc l'avoir menée à son achèvement) et l'avoir encore. On ne peut avoir mené une action praxique à son achèvement et l'avoir encore.

/7 ibid., p. 56. L'exploration comme une suite de révisions qui enchaîne des actions et l'utilisation qui consomme le résultat d'une de ces actions. 
/8 Voir les deux questions posées à Hans Belting par Umut Ungan, p. 17 de ce numéro.

19 Base de documentation sur la musique contemporaine, [http://brahms.ircam. fr/works/work/8977], consulté le 19 mai 20 pluriel permettant une pluralité d'usage. Par exemple, un usage socio-politique pour l'œuvre de Satie; un usage fictionnel pour l'œuvre de Tinguely; un usage vernaculaire où le temps de l'œuvre s'initie et pourrait se développer dans notre quotidienneté (Warhol, Christian Marclay)...

Un deuxième cadre serait l'approche de Hans Belting, et plus particulièrement ce qu'il nous dit dans ses réponses aux deux questions qui lui ont été adressées dans ce numéro. Il s'agit ici d'envisager une diversité de "récit », de " discours » des disciplines qui font le monde de l'art et une interaction, des jeux "fictionnels » et "narratifs " entre eux. Hans Belting nous propose d'élargir le temps historique de l'art en passant «du vertical (histoire) à l'horizontal (espace)/8 ». Ce qui peut conduire à des approches anachroniques dont celle " anthropologique des images ». Parallèlement, il pose la question du temps qui a été celle d'une histoire progressiste de l'art, et qui est elle-même située dans le temps. Ainsi en déconstruisant et situant historiquement la vision téléologique de cette histoire, la posture de Belting permet d'inscrire les images contemporaines, qu'elles soient artistiques ou pas, dans la longue histoire des images.

La multiplicité, en ce qui concerne les angles d'approche et les objets étudiés par les auteurs de la journée d'études, rend compte de la pluralité, mais aussi de la complexité de la question des temps qui traversent les œuvres et qui les constituent, qu'elles soient cinématographiques, musicales ou encore plastiques.

\section{Abstraction et multiplicité des temps : exemple de Vortex Temporum}

Nous avons aussi relevé un développement artistique de cette pluralité temporelle dans certaines nouvelles recherches chorégraphiques associant plasticité et musicalité afin de déconstruire l'opposition traditionnelle entre les arts de l'espace et ceux du temps. Ainsi, il arrive que les différentes disciplines artistiques dialoguent autour de la question du temps en proposant des formes temporelles hybrides. Vortex Temporum, œuvre chorégraphique d'Anne Theresa De Keersmaeker, en est un parfait exemple. L'œuvre de la chorégraphe belge puise directement dans celle, éponyme, du musicien français Gérard Grisey composée entre 1994 et 1996. Ce dernier parle, à propos de Vortex Temporum (Tourbillon de Temps), de la «naissance d'une formule d'arpèges tournoyants et répétés et sa métamorphose dans différents champs temporels ", selon une démarche qui s'inscrit dans la volonté "d'abolir le matériau au profit de la durée pure/9 ". Ecrite pour six instruments (piano, violoncelle, alto, violon, clarinette, 
flûte), cette musique de chambre en trois mouvements est prise comme objet par la chorégraphe belge qui n'en est pas à son premier rapprochement avec la musique (elle a en effet déjà travaillé autour de l'œuvre de Miles Davis, de Steve Reich ou encore de Gustave Mahler). Si l'œuvre musicale désire "abolir le matériau» par mouvement " tourbillonnaire » des notes et des sons modulés, autrement dit les inscrire dans la durée, comment une œuvre chorégraphique peut rendre cela " gestuel »?

C'est derrière cette volonté "métaphysique » de la transmissibilité d'un message pur de Grisey que va se jouer cette conversation entre la danse et la musique, où le dépouillement des langages vont amener à se questionner sur et à travers les différentes temporalités à l'œuvre. Il est important de souligner le fait qu'il ne s'agit pas ici d'une simple transposition ni d'accompagnement concernant la musique. D'une manière générale, on peut affirmer que le dialogue entre ces deux pratiques contemporaines est plutôt coutumier, dû notamment à la démarche déconstructionniste de leurs propres modèles historiques et de l'élargissement des codes respectifs: dans le cas de la danse contemporaine, il s'agissait des expressions corporelles qui jusquelà n'avaient pas un statut artistique et qui se sont insérées dans le champ chorégraphique; de même pour des sonorités étrangères aux règles de l'harmonie et de la composition qui se voyaient légitimées dans et par la musique contemporaine. Ce processus a eu pour corollaire un degré d'abstraction élevé quant aux formes, qu'elles relèvent de la gestualité dans le cas de la danse ou qu'elles soient compositionnelles concernant la musique, créant ainsi une lisibilité et surtout une «traductibilité » des structures ontologiquement différentes. La traductibilité est ici à entendre selon un sens interprétatif, puisque les deux « systèmes » sémiotiques que peuvent incarner la danse et la musique contemporaine, même si elles peuvent être considérées comme des langages, ne sont pas transposables sur le plan textuel, ni ne possèdent une charge expressive inhérente.

L'abstraction facilite donc d'une certaine manière le dialogue en permettant un investissement plus important des signes. Vortex Temporum de Keersmaeker peut ainsi prétendre à « mettre en geste » la musicalité propre à la pièce de Grisey, et c'est autour d'un lieu commun que la chorégraphe va pouvoir élaborer son œuvre. Ce lieu commun est celui d'un langage épuré et formel, où les différentes temporalités vont pouvoir se joindre et penser ensemble. Par exemple, pour déjouer la discontinuité temporelle au niveau attentionnel que pourrait exiger toute représentation, Keersmaeker fait entrer les musiciens qui commencent l'exécution de l'œuvre de Grisey avant même l'extinction des lumières de la salle. Le spectateur habitué à 
/10 Dans le sens de Francis Jacques. Voir Francis Jacques, L'Espace logique de l'interlocution, Paris, PUF, 1985.

une délimitation classique du temps et de l'espace représentationnel, sans indication du commencement du spectacle, se trouve ainsi surpris par cet « irruption » de l'œuvre. Ce mélange temporel s'active également sur scène entre les musiciens et les danseurs: ces derniers au départ distingués, se mélangent dans les parties ultérieures du spectacle ; les musiciens, tout en continuant à jouer, deviennent mobiles et réalisent les mouvements circulaires en compagnie des danseurs. Composé à la manière d'un tableau, les différences de traits et de fonctions sur scène s'estompent et se confondent. On se retrouve ici avec un réel jeu des temporalités différentes: celui entre le temps du public et de la performance sonore; entre le rythme variant parmi les danseurs; entre les musiciens et les danseurs; entre les musiciens/danseurs et les danseurs. Tout ce travail autour des différentes temporalités s'inscrit naturellement sur le plan de l'intentionnalité artistique : la représentation étant « totale », cette stratification temporelle n'est pas un élément a priori nécessaire pour apprécier l'œuvre. Or c'est cette « polyphonie », si l'on peut reprendre un terme utilisé aussi bien en musique qu'en théorie de la littérature, qui enrichit l'expérience esthétique.

Ne peut-on aller plus loin en envisageant que les œuvres qui engendrent une pluralité temporelle de leur production, de leur manifestation jusqu'à leur activation, puissent permettre de passer de polyphonie/polysémie à une plurivocité/10 ? Ces interactions créatives entre un passé, un présent et un futur de l'œuvre ne conduisent-elles pas à un mode de « dialogisme temporel », si non intersubjectif du moins inter/ trans-temporel? Le pluralisme temporel d'une œuvre n'engage-t-il pas à un dialogisme sur et par le temps, à « plurivocité temporelle »?

\section{Stéphane Reboul et Umut Ungan}

